\title{
Estudo das vias biliares em muares (Equus asinus $X$ Equus caballus). Sistematização do ductus principalis sinister
}

\section{Study of the biliar ducts in mules (Equus asinus $X$ Equus caballus). Sistematization of the ductus principalis sinister}

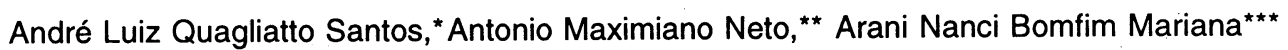

\begin{abstract}
Resumo
Para realização deste trabalho, utilizaram-se 30 fígados, provenientes de muares adultos, de diferentes e não conhecidas idades, sendo 15 machos e 15 fêmeas, oriundos do Frigorífico Pomar Ltda, no município de Araguari, estado de Minas Gerais. Injetaram-se as vias biliares, esvaziadas o quanto possível, mediante suaves e sucessivas massagens, com Neopreme Latex 450 (Du Pont do Brasil S/A Indústrias Químicas) acrescida de Celobar (Laboratórios Maurício Villela), em suspensão a 50\%. Após a sua fixação em solução aquosa de formol a $10 \%$, por 48 horas, promoveu-se a sua dissecação, com pinças e tesouras cirúrgicas. Verificou-se que o sistema do ductus principalis sinister é composto pelo ductus ventralis lobi sinistri lateralis, ductus medius lobi sinistri lateralis, ductus lobi sinistri medialis, registrados em todas as preparações (100,0\%), pelo ductus dorsalis lobi sinistri lateralis $(96,7 \%)$, ductus lobi quadradi $(80,0 \%)$ e por coletores inominados provenientes do lobus sinister lateralis (territórios dorsal, médio e ventral), lobus sinister medialis e lobus quadratus.
\end{abstract}

Palavras-chave: eqüideos, fígado, anatomia.

\begin{abstract}
In the present study, thirty livers from adult mules, 15 male and 15 female, with different and unknown ages, were obtained from the Pomar Ltda slaughterhouse in Araguari, MG. The bile ducts were injected with Neoprene latex 450 added of a $50 \%$ Celobar suspension after have been emptied by slight and successive massages. Next, the livers were fixed in a $10 \%$ formol aqueous solution for 48 hours and then submitted to dissection. The ductus principalis sinister system was constituted of the ductus ventralis lobi sinistri lateralis, the ductus medius lobi sinistri lateralis, the ductus lobi sinistri medialis (which were found in all preparations), the ductus dorsalis lobi sinistri lateralis $(96.7 \%)$, the ductus lobi quadradi $(80.0 \%)$ and the unnamed collectors coming from the lobus sinister lateralis (ventral, medium and dorsal territories), the lobus sinister medialis and the lobus quadratus.
\end{abstract}

Keywords: equines, liver, anatomy.

\section{Introdução}

O fígado de mamíferos domésticos representa, do ponto de vista funcional, o local de diversos processos metabólicos, pois recebe todo o material absorvido ao nível de intestinos, exceção feita a certa quantidade de lipídeos transportados por via linfática. Devido a essas importantes mediações, o órgão apresenta-se em situação de grande destaque no organismo, já que metaboliza, armazena, sintetiza e elimina substâncias absorvidas. Tal fato se processa pela bile, secreção exócrina das células hepáticas, de grande importância na digestão de gorduras. Devido às mencionadas características, numerosos são os pesquisadores ocupados em estudar os múltiplos aspectos macroscópicos desta complexa glândula, especialmente quando nos reportamos ao seu sistema excretor.
Considerando também a escassez de informações na literatura a respeito deste assunto, propomos, além de completála, contribuir também para o desenvolvimento da anatomia comparativa, especialmente entre os eqüídeos.

De maneira geral, Chauveau e Arloing (1903), Berg (1978) e Dyce et al. (1987) descrevem, para os eqüinos, apenas os limites cranial e caudal, diafragma e extremidade da $17^{\mathfrak{a}}$ costela, respectivamente e a ausência de vesícula biliar.

Kraehmer e Schröder (1976) citam que os equinos têm 3/5 do seu fígado na parte direita do plano mediano, a porção direita está dirigida dorsalmente e a esquerda, que é menor, encontra-se ventralmente.

Frandson (1979), D'Arce e Flechtmann (1989) comentam que todos os mamíferos domésticos, com exceção do cavalo,

\footnotetext{
* Professor titular da Faculdade de Medicina Veterinária da Universidade Federal de Uberlândia. Av. Ceará s/nº, Campus Umuarama, Uberlândia, MG. CEP 38400-902.

** Professor substituto da Universidade de Brasília.

*** Professora doutora da Faculdade de Medicina Veterinária e Zootecnia da Universidade de São Paulo.
} 
possuem vesícula biliar, que pode apresentar-se como mera dilatação do ducto hepático ou, então, ligar-se a este através do ducto cístico para formar o chamado ducto biliar comum.

Schwarze e Schröder (1970) relatam a presença do lobus sinister lateralis, lobus sinister medialis, lobus dexter, lobus quadratus e lobus caudatus.

Caradonna (1930) e Getty (1986) citam que o ducto hepático é formado pela união dos ductos hepáticos direito e esquerdo.

Nusshag (1977) comenta que, nos animais domésticos, a secreção da bile é constante e está armazenada na vesícula biliar (exceto no cavalo que não a possui).

Jablan-Pantic (1963), após a injeção dos fígados com substância radiopaca, através do ductus coledocus, seguida de fixação pela formalina a $4 \%$, concluiu que:

a) o ductus hepaticus, cuja secção distal representa o ductus coledocus, fornece dois ramos, isto é, o ramus principalis dexter e o ramus principalis sinister,

b) o ramus principalis dexter drena o lobus dexter mais o processus caudatus, por intermédio de um ramo ventral e de três a cinco ramos laterais e dorsais - rami lobi dextri, servidos às porções homônimas daquele lobo, após o que penetra no lobo caudato, como ramus processi caudati;

c) o ramus principalis sinistri dá vazão à bile do lobus sinister lateralis, do lobus sinister medialis, do lobus quadratus e da porção inicial do lobus caudatus, por meio de dois rami dorsalis lobi sinistri lateralis, quatro rami medii lobi sinistri lateralis, dois rami ventralis lobi sinistri lateralis, dois ou três rami lobi sinistri medialis, um ou dois rami lobi quadrati e coletor inominado do lobus caudatus.

Bradley (1922), indica um dos ductos hepáticos, o mais conspícuo, como responsável pela drenagem dos lobos lateral e central esquerdos; o outro serve ao lobo lateral direito. Ducto biliar e ducto colédoco são sinônimos, para o citado autor.

Bourdelle e Bressou (1937) descrevem que as vias biliares correm para a porta hepatis, onde se localizam no canal do colédoco, em relação aos eqüídeos.

Em comentário sobre as vias biliares extra-hepáticas, Bruni e Zimmerl (1947) atribuem à via bilífera do lobo médio a abrirse na volumosa raiz esquerda do conduto hepático.

Ellenberger e Baum (1932) apontam a existência de dois troncos confluentes, direito e esquerdo, o último deles, grosso, achado a acolher o lobo médio. Constituído o ductus hepaticus, aplicam ao seu segmento terminal a designação de ductus coledocus.

Já Favilli (1923) e Gonzalez Y Garcia e Gonzalez Alvarez (1961), divergem dos autores precedentes apenas no que diz respeito à denominação do ductus coledocus, pois, relatam, respectivamente, como hepato-entérico e conduto colédoco.

O desenho citado no estudo de Duprat (1970) serve como parâmetro para um modelo de distribuição, cujos ramos seriam, por ordem, o ductus medius lobi sinistri lateralis mais o ductus ventralis lobi sinistri lateralis, o ductus dorsalis lobi sinistri lateralis, o ductus lobi sinistri medialis, e o ductus lobi quadrati.

Nickel et al. (1973) demarcam o fígado por dois planos imaginários, perpendiculares às superfícies diafragmática e visceral do órgão. Um deles acompanha o maior eixo da fissura hepática direita até atingir, dorsalmente, o sulco da veia cava caudal; o outro percorre a incisura correspondente ao ligamento redondo e à impressão esofágica.

Quanto à literatura especializada, alusiva ao focado assunto, cabe destacar os trabalhos realizados por Ferreira (1974), a respeito das vias bilíferas intra e extra-hepáticas de eqüinos sem raça definida, por Stopiglia (1981), sobre o comportamento do ductus coledocus, além da sistematização dos tributários do ductus principalis dexter no Puro-Sangue Inglês e, mais recentemente, por Marçal et al. (1993), com a sistematização do ductus principalis sinister no Puro-Sangue Inglês, os quais evidenciaram a importância de se dar continuidade àquela série de trabalhos.

Além disso, destacamos as observações de Ottaviani (1933), em órgãos de animais adultos, onde o ducto biliar esquerdo é resultante da associação de dois afluentes primários e identificado a escoar a bile dos lobos esquerdo, quadrado e caudado, mediante variado número de afluentes primários, um deles endereçando-se ao segmento inicial do colédoco. Já Duprat (1970) propõe um modelo de distribuição das vias bilíferas, onde o fígado do cavalo é dividido nos segmentos por ele denominados de I, II, III, IV e IV'.

\section{Material e métodos}

Utilizamos 30 peças, compreendendo, cada uma, em bloco, fígado e o segmento intestinal que contém a ampola hepatopancreática, pertencentes a muares adultos, de diferentes $\mathrm{e}$ não conhecidas idades, sendo 15 machos e 15 fêmeas, provenientes do Frigorífico Pomar Ltda. no município de Araguari, estado Minas Gerais.

Aberto o duodeno, ao longo de sua borda livre, e exposta a mucosa, canalizou-se o ductus coledocus, através da ampola hepato-pancreática, e injetaram-se as vias biliares, esvaziadas quanto possível, mediante suaves e sucessivas massagens, com Neopreme Latex 450 (Du Pont do Brasil S/A. Indústrias Químicas), acrescido de Celobar (Laboratórios Maurício Villela), em suspensão a 50\%. Após a sua fixação em solução aquosa de formol a $10 \%$ por 48 horas, promoveu-se a sua dissecação, com pinças e tesouras cirúrgicas.

A título de ilustração do trabalho, foram realizadas radiografias, sendo estas fotografadas (Figura 1). Elaboraram-se, também, desenhos esquemáticos, representativos da distribuição dos ductos biliares, mais precisamente sobre o ductus principalis sinister, em fígados de muares (Figuras $2-32$ ).

Visando facilitar a descrição dos resultados, e como os lobos hepáticos nos muares apresentam-se parcialmente limitados por incisuras, demarcou-se o fígado conforme a proposição de Nickel et al. (1973), por dois planos imaginários, perpendiculares às superfícies diafragmática e visceral do órgão. Um deles acompanhando o maior eixo da fissura hepática direita até atingir, dorsalmente, o sulco da veia cava caudal; o outro percorrendo a incisura correspondente ao ligamento redondo e à impressão esofágica.

Assim, à direita do primeiro localiza-se o lobus dexter, pequena parte do lobus caudatus e o processi caudati e, à esquerda do segundo, o lobus sinister lateralis e o lobus sinister medialis. No segmento intermédio, encontra-se, ventralmente, o lobus quadratus e dorsalmente a porção inicial do lobus caudatus (pars supraportalis). 
Figura 1 - Radiografia de fígado de muar, mostrando o arranjo das vias biliares componentes do ductus principalis sinister

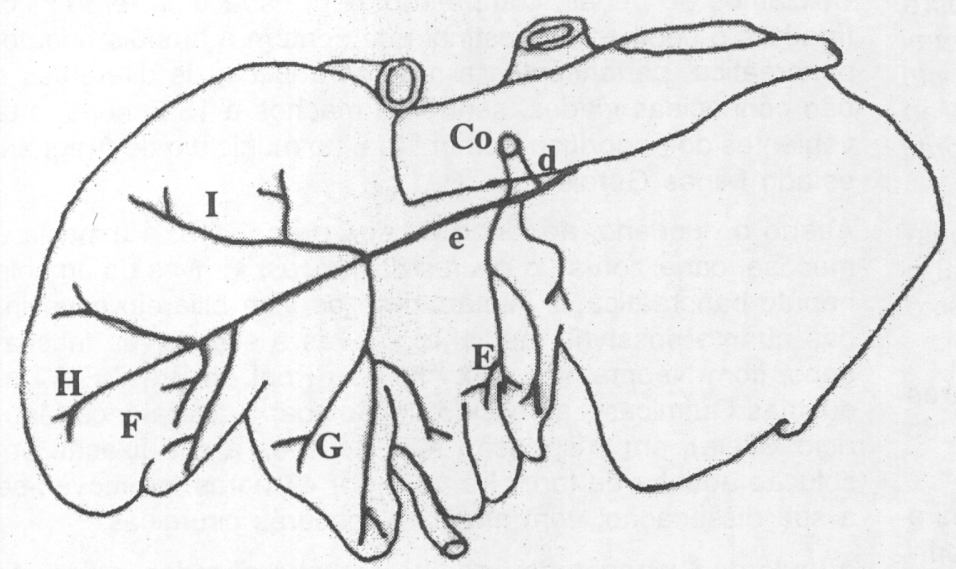

Figuras 1 e 2

E - ductus lobi quadrati

F - ductus ventralis lobi sinistri lateralis

G - ductus lobi sinistri medialis

$\mathrm{H}$ - ductus medius lobi sinistri lateralis

I - ductus dorsalis lobi sinistri lateralis

d - ductus principalis dexter

e - ductus principalis sinister

Co - ductus choledocus

Figura 2 - Desenho esquemático, representativo do padrão de distribuição do ductus principalis sinister

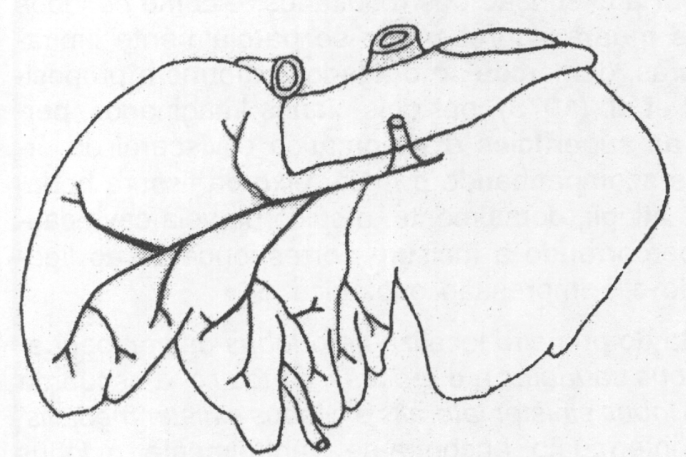

Figura 3 - Macho

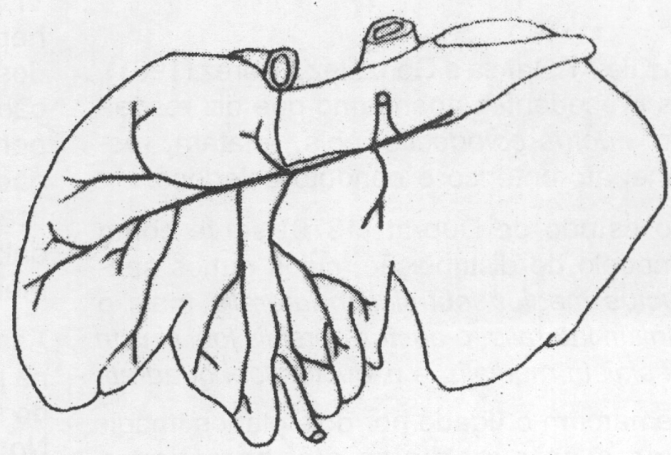

Figura 4 - Macho 


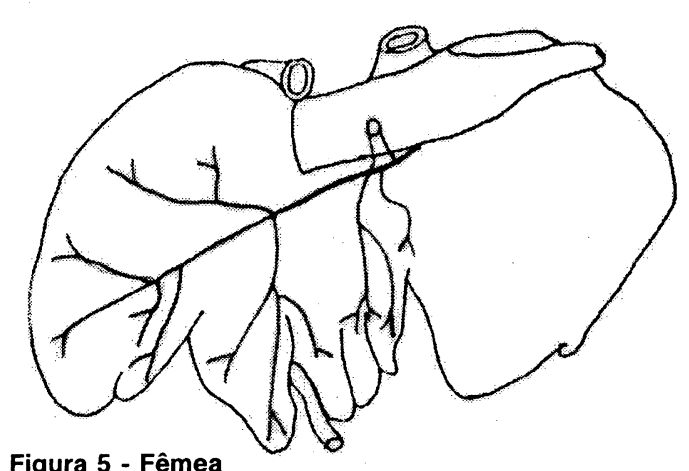

Figura 5 - Fêmea

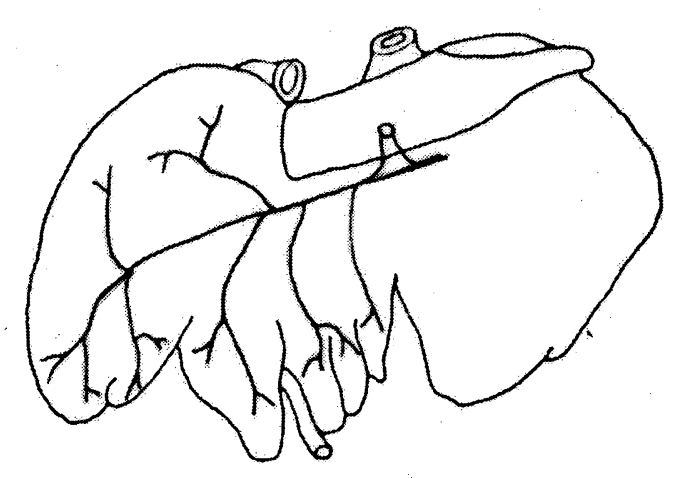

Figura 7 - Fêmea

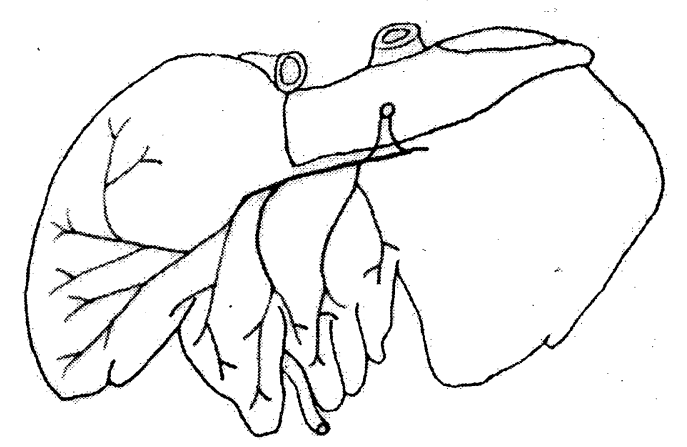

Figura 9 - Macho

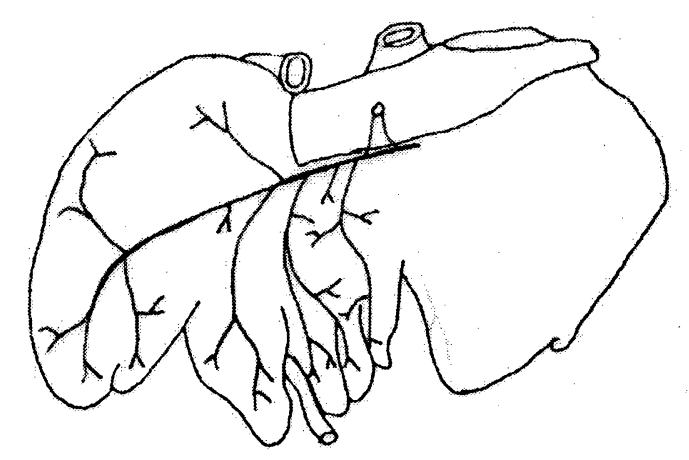

Figura 11 - Fêmea

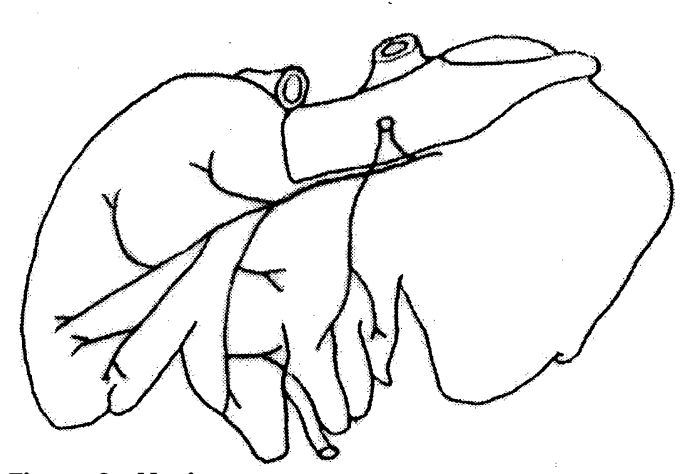

Figura 6 - Macho

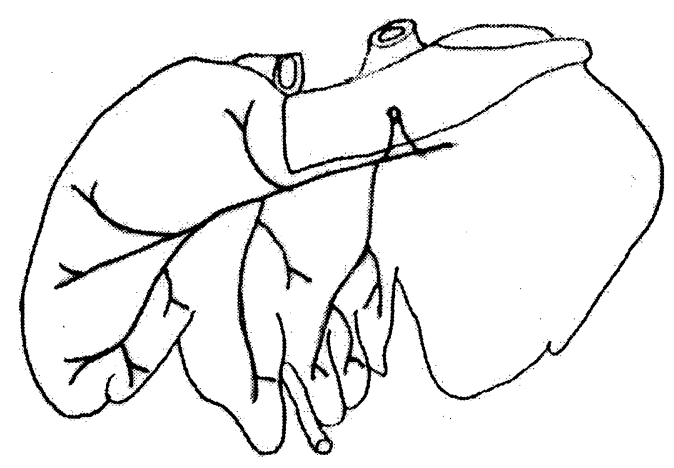

Figura 8 - Macho

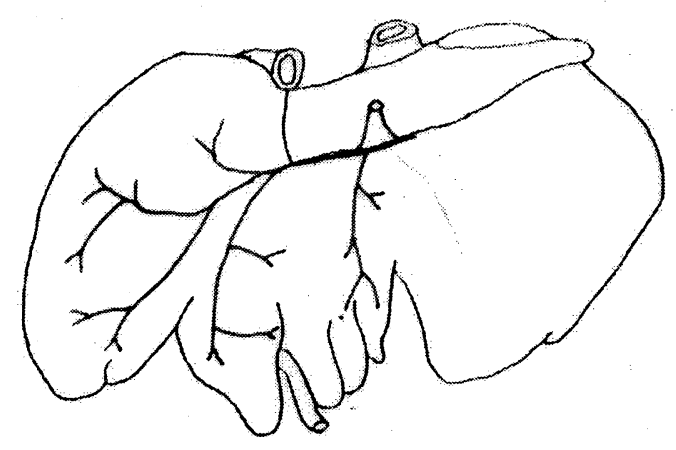

Figura 10 - Macho

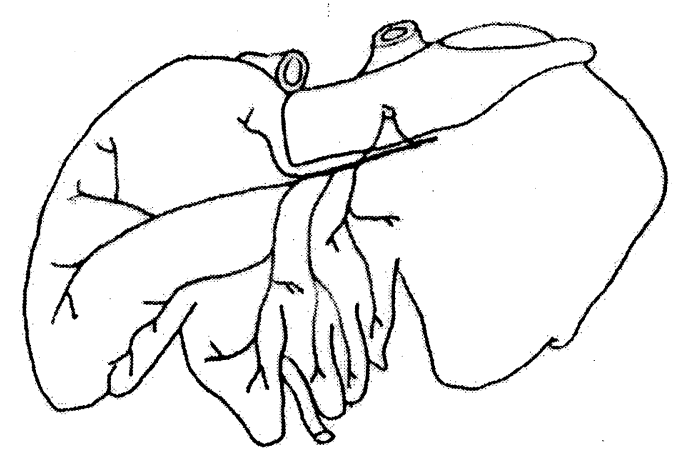

Figura 12 - Fêmea 


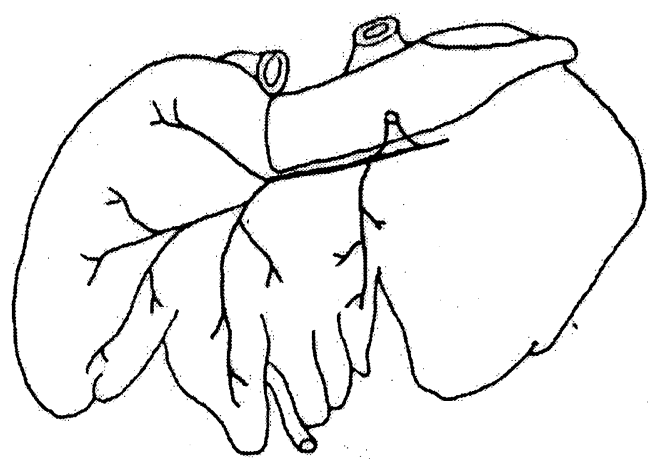

Figura 13 - Macho

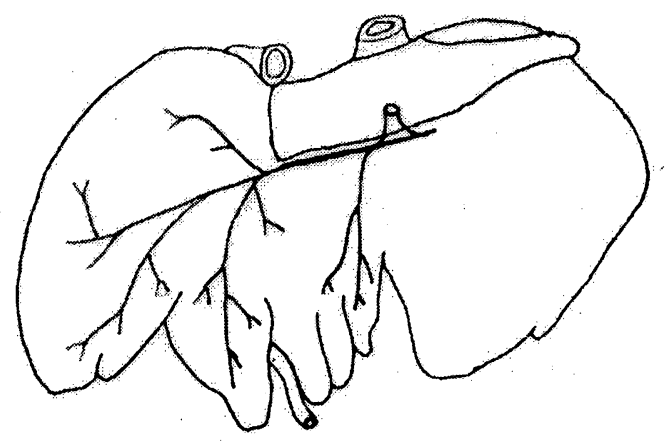

Figura 15 Fêmea

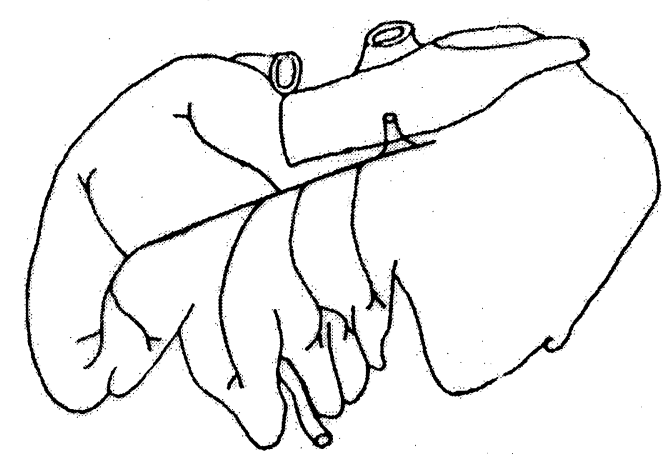

Figura 17 - Fêmea

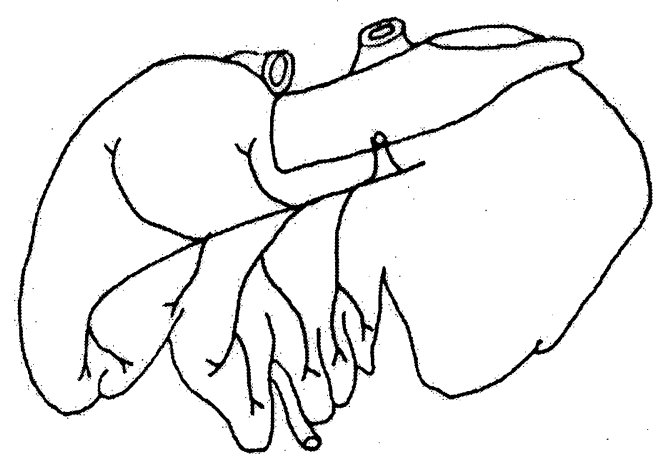

Figura 19 - Macho

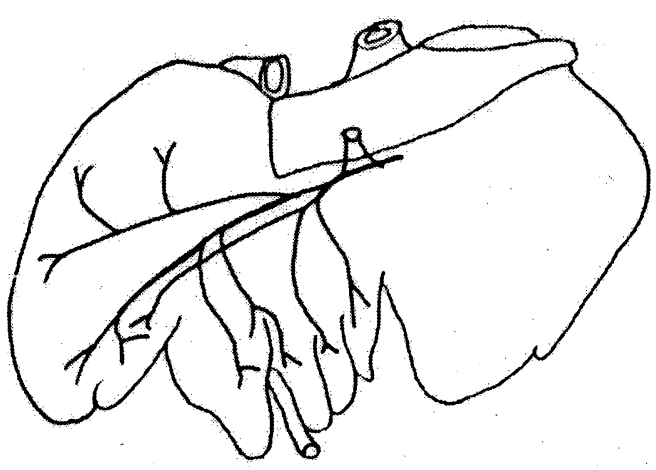

Figura 14 - Fêmea

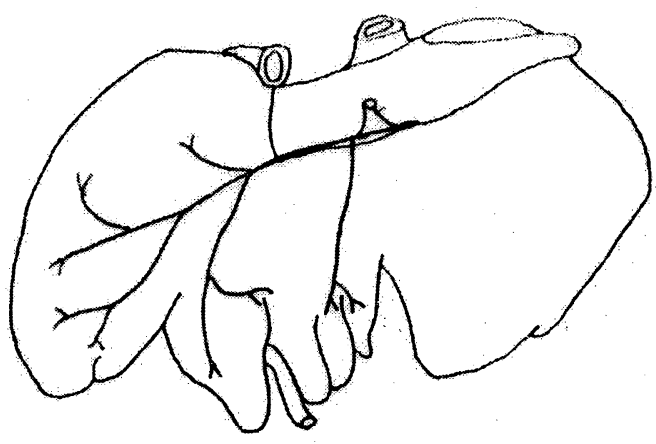

Figura 16 - Fêmea

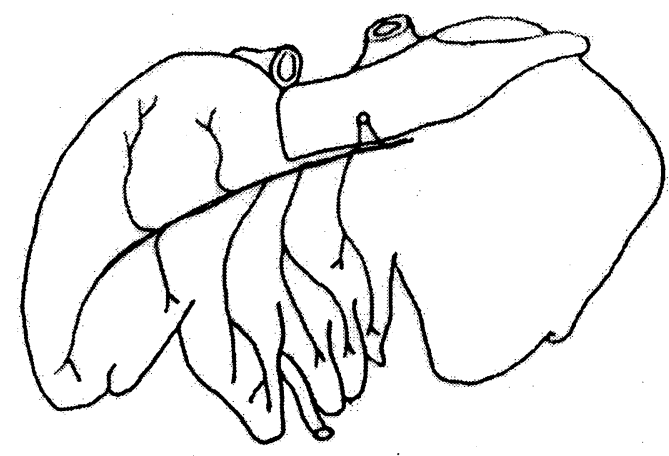

Figura 18 - Fêmea

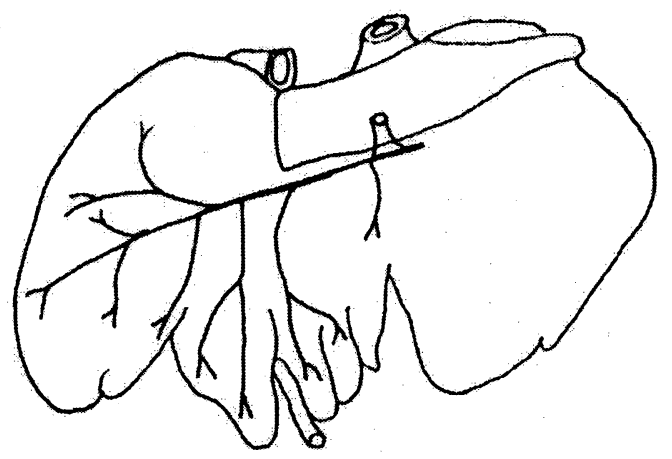

Figura 20 - Macho 


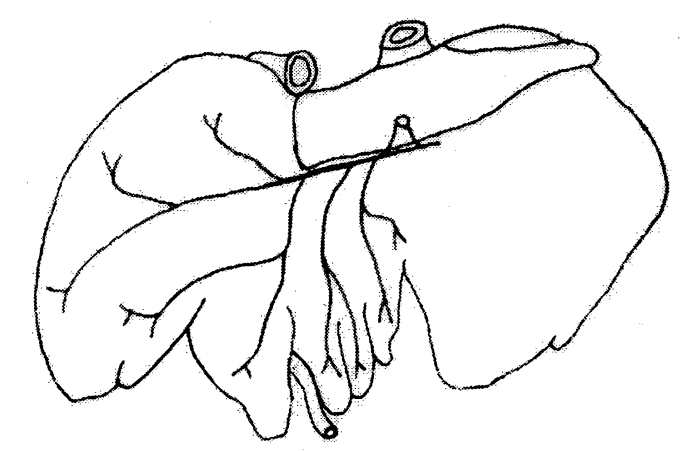

Figura 21 - Fêmea

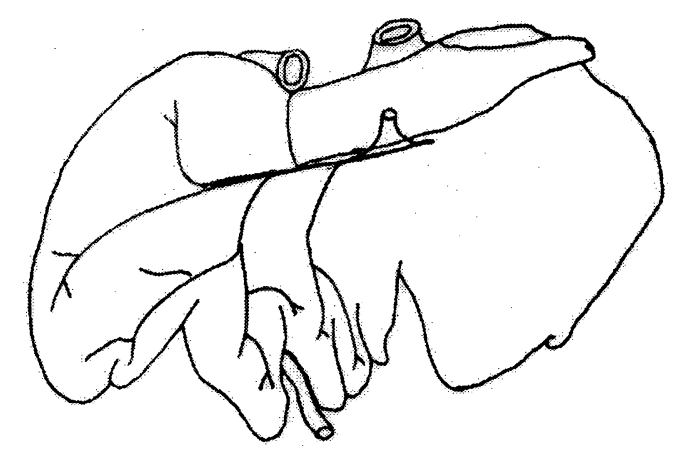

Figura 23 - Fêmea

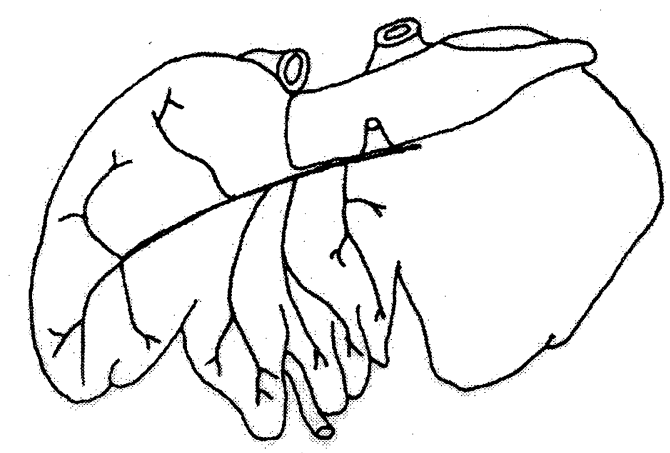

Figura 25 - Fêmea

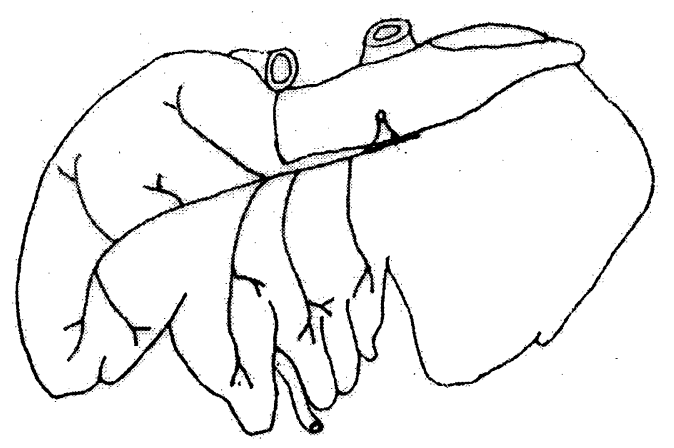

Figura 27 - Fêmea

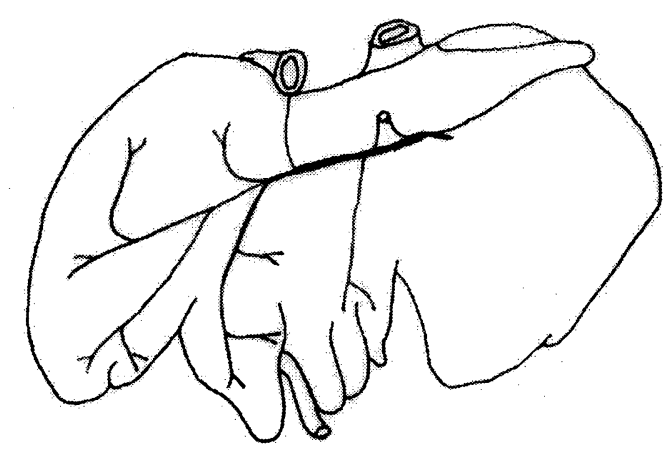

Figura 22 - Macho

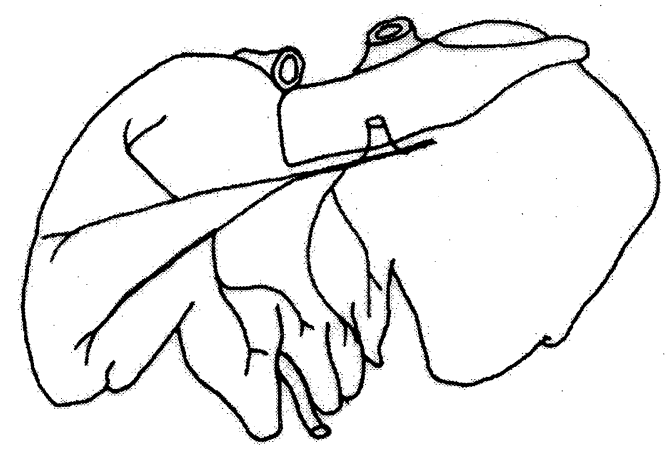

Figura 24 - Fêmea

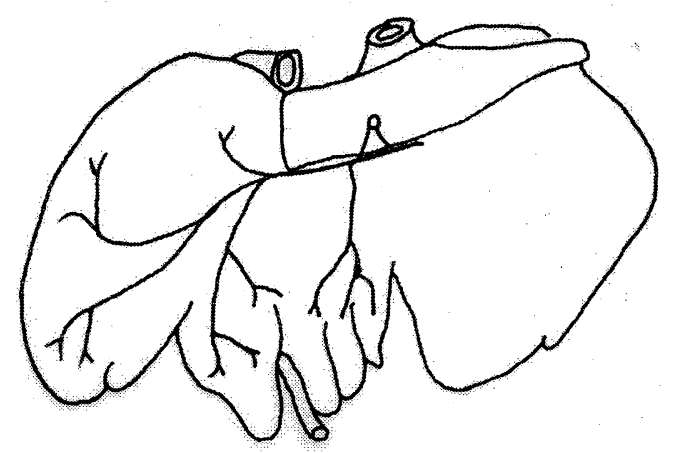

Figura 26 - Macho

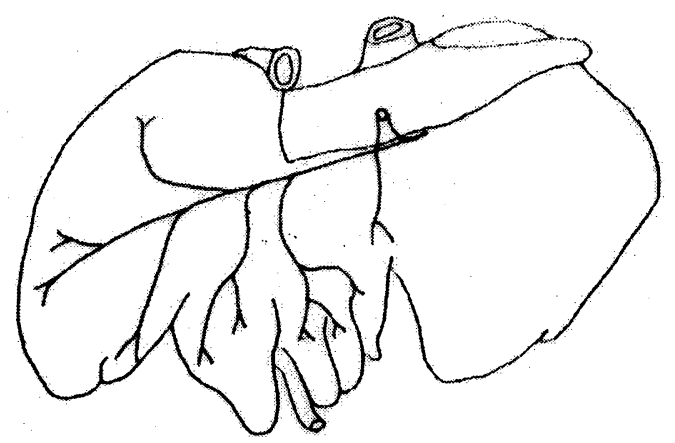

Figura 28 - Macho 


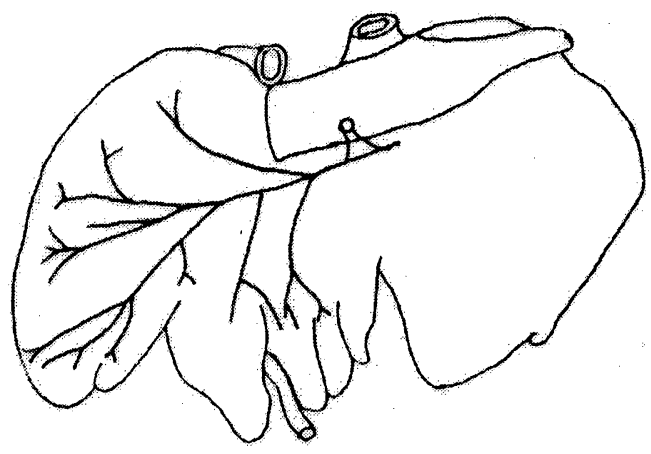

Figura 29 - Fêmea

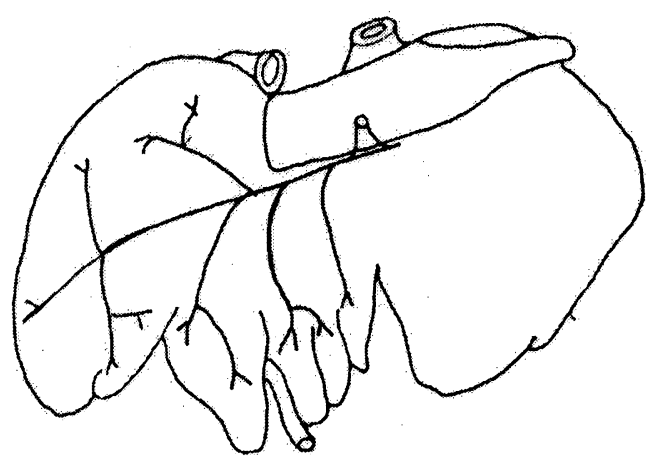

Figura 31 - Macho

Finalmente, empregou-se a nomenclatura modificada adotada por Jablan-Pantic (1963), bem como a recomendada pelo International Committee on Veterinary Gross Anatomical Nomenclature (1994).

\section{Resultados}

O sistema do ductus principalis sinister é composto pelo ductus ventralis lobi sinistri lateralis, ductus medius lobi sinistri lateralis, ductus lobi sinistri medialis, registrado em todas as preparações ora estudadas, ductus dorsalis lobi sinistri lateralis (96,7\%), ductus lobi quadrati $(80,0 \%)$ e coletores inominados provenientes do lobus sinister lateralis (territórios dorsal, médio e ventral), lobus sinister medialis, lobus quadratus.

Uma vez individualizado, o ductus principalis sinister apresenta-se livre de tributários em seis dos 30 fígados $(20 \%)$ acoIhendo, nos outros $24(80 \%)$, contingentes procedentes exclusivamente do lobus quadratus.

Encontrou-se, para o ductus principalis sinister, número variável de raízes que, em sucessivos arranjos, compõem as seguintes vias: o ductus medius lobi sinistri lateralis mais ductus ventralis lobi sinistri lateralis, ductus lobi sinistri medialis, o ductus dorsalis lobi sinistri lateralis e o ductus lobi quadrati (26,7\%); o ductus medius lobi sinistri lateralis mais o ductus ventralis lobi sinistri lateralis, o ductus lobi sinistri medialis, o ductus dorsalis lobi sinistri lateralis (10\%); o ductus medius lobi sinistri lateralis mais ductus ventralis lobi sinistri lateralis, o ductus dorsalis lobi sinistri lateralis, o ductus lobi sinistri medialis e o ductus lobi quadrati (30\%); o ductus medius lobi sinistri lateralis mais ductus ventralis lobi sinistri lateralis,

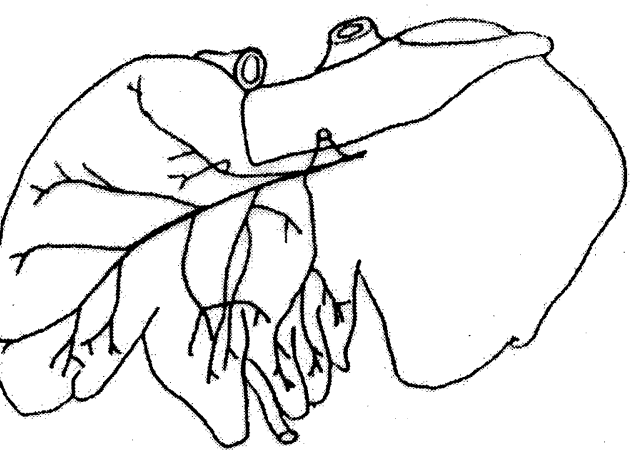

Figura 30 - Macho

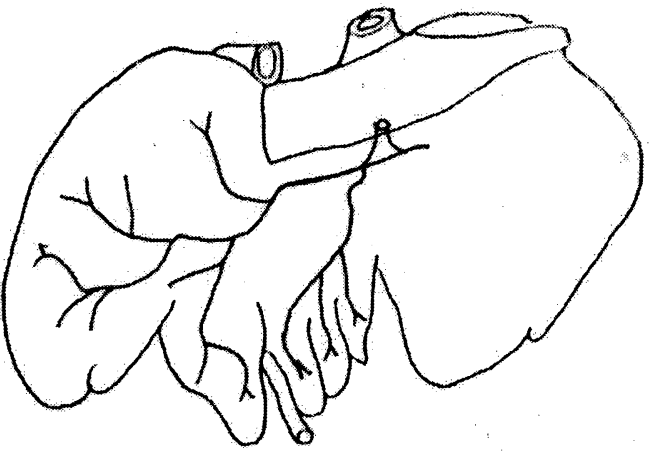

Figura 32 - Macho

ductus dorsalis lobi sinistri lateralis, ductus lobi sinistri medialis (3,3\%); o ductus medius lobi sinistri lateralis mais ductus ventralis lobi sinistri lateralis, ductus lobi sinistri medialis e 0 ductus lobi quadrati (6,7\%); o ductus medius lobi sinistri lateralis mais ductus ventralis lobi sinistri lateralis, ductus lobi sinistri medialis, ductus lobi quadrati (10\%); o ductus medius lobi sinistri lateralis mais ductus ventralis lobi sinistri lateralis, ductus lobi sinistri medialis, ductus dorsalis lobi sinistri lateralis, ductus lobi quadrati (3,3\%); o ductus medius lobi sinistri lateralis mais o ductus ventralis lobi sinistri lateralis, ductus lobi sinistri medialis, ductus lobi quadrati, ductus dorsalis lobi sinistri lateralis $(3,3 \%)$.

O ductus dorsalis lobi sinistri lateralis, ductus medius lobi sinistri lateralis, ductus ventralis lobi sinistri lateralis, o ductus lobi sinistri medialis e o ductus lobi quadrati drenam como ductos conspícuos, respectivamente, os territórios dorsal, médio e ventral do lobus sinister lateralis, o lobus sinister medialis e o lobus quadratus, neles ou nas vias comuns para os quais concorrem, desembocam inúmeros afluentes inominados vindos das mencionadas regiões glandulares.

\section{Discussão}

No concernente à sistematização do ductus principalis sinister, identificou-se o mesmo, de forma geral, a drenar o lobus sinister lateralis (territórios dorsal, médio e ventral), lobus sinister medialis, e lobus quadratus.

Para Ferreira (1974) e Marçal et al. (1993), cujas conclusões revestem-se de maior importância, o ductus principalis sinister acolhe a bile dos mesmos territórios indicados para os mu- 
ares, com exceção feita ao aferente inominado do processus caudatus do lobus caudatus, mencionado por Ferreira (1974).

Ao seu turno, Jablan-Pantic (1963) aponta o ductus principalis sinister recebendo a bile do lobus sinister lateralis, lobus sinister medialis, lobus quadratus e parte inicial do lobus caudatus, por meio, sucessivamente, de dois rami dorsalis lobi sinistri lateralis, quatro rami medii lobi sinistri lateralis, dois ou três rami lobi sinistri medialis, um ou dois rami lobi quadrati e contingentes do lobus caudatus.

Conforme observou-se nos muares, bem como para Ferreira (1974) e Marçal et al. (1993), todos os ductos relacionados por Jablan-Pantic (1963), afora outros, foram assinalados, cabendo esclarecer todavia que, neste estudo, foram visualizados já formados, em razão da convergência de suas raízes e de cuja destinação, diversa na configuração geral das vias biliares, será tratada adiante.

No entender de Ottaviani (1933), em órgãos de animais adultos o ducto biliar esquerdo (ductus principalis sinister) é resultante da associação de dois afluentes primários e identificado a escoar a bile dos lobos esquerdo, quadrado e caudado (setor esquerdo), mediante número diverso de aferentes primários, um deles endereçando-se ao segmento inicial do colédoco.

Comparando as observações realizadas nos muares, com as de Ferreira (1974) para o Equus caballus e as de Marçal et al. (1993) para o eqüino da raça Puro-Sangue Inglês, incluindo a radiografia constante de seu trabalho, entendeu-se que os dois aludidos afluentes primários, componentes do ducto biliar esquerdo, representam troncos, por ordem, o ductus dorsalis lobi sinistri lateralis mais ductus lobi sinistri medialis ou ductus medius lobi sinistri lateralis mais ductus ventralis lobi sinistri lateralis. Provavelmente, os demais ramos principais correspondem ao ductus lobi quadrati e a contingentes inominados do lobus sinister lateralis, lobus sinister medialis, lobus quadratus e lobus caudatus.

$\mathrm{Na}$ fotografia constante da pesquisa de Jablan-Pantic (1963), observou-se que 0 ductus principalis sinister mostra-se individualizado, pela composição, obedecido o sentido periferiacentro, do ductus medius lobi sinistri lateralis mais ductus ventralis lobi sinistri lateralis, ductus lobi sinistri medialis, ductus dorsalis lobi sinistri lateralis e ductus lobi quadrati, arranjo também encontrado por Ferreira (1974) e Marçal et al. (1993), em $53,3 \%$ e $36,7 \%$, respectivamente, do material pesquisado, e agora para os muares, em $30 \%$. Segundo Duprat (1970), o ductus hepaticus sinister (ductus principalis sinister) drena o lobus sinister lateralis (segmento II) e recebe afluentes dorsais e ventrais da pars supraportalis do lobus caudatus (segmento I), do ductus sinister medialis (segmento III) e do lobus quadratus (segmentos IV e IV').

Focalizando o ductus principalis sinister, depois de individualizado, Ferreira (1974) o encontrou livre de afluentes em 15 das 30 preparações $(50 \%)$ e a receber tributários nas outras $15(50 \%)$, estes procedentes: apenas do lobus quadratus $(16,7 \%)$; simultaneamente do lobus quadratus e lobus dexter (território ventral) (13,3\%); conjuntamente do lobus sinister lateralis (território dorsal) e lobus quadratus (6,7\%); somente do lobus dexter (território ventral) (3,3\%); concomitantemente do lobus quadratus, lobus dexter (territórios médio e ventral) e lobus caudatus $(3,3 \%)$; simultaneamente do lobus sinister lateralis (território dorsal), lobus quadratus, lobus dexter (ter- ritório ventral) e lobus caudatus (3,3\%). Já nas dissecações de Marçal et al. (1993), o ductus principalis sinister, evidenciado livre de aferentes em 13 dos 30 fígados (43,3\%) acolhe, nos demais $17(56,7 \%)$, contingentes oriundos: simultaneamente do lobus quadratus e lobus caudatus (pars supraportalis) (13,3\%); exclusivamente do lobus quadratus (10\%); concomitantemente do lobus quadratus e lobus dexter (território ventral) (10\%); apenas do lobus caudatus (pars supraportalis) $(56,7 \%)$; contingentes oriundos: simultaneamente do lobus quadratus e lobus caudatus (pars supraportalis) (13,3\%); exclusivamente do lobus quadratus (10\%);concomitantemente do lobus quadratus e lobus dexter (território ventral) $(10,0 \%)$; apenas do lobus caudatus (pars supraportalis) (6,7\%); somente do lobus dexter (território ventral) $(3,3 \%)$; simultaneamente do lobus quadratus, lobus caudatus (pars supraportalis) e lobus sinister lateralis (território ventral) $(3,3 \%)$; concomitantemente do lobus quadratus, lobus sinister medialis e lobus caudatus (pars supraportalis) $(3,3 \%)$; simultaneamente do lobus quadratus, lobus caudatus (pars supraportalis), lobus sinister medialis e lobus sinister lateralis (território dorsal) (3,3\%); conjuntamente do lobus caudatus (pars supraportalis), lobus quadratus, lobus dexter (território ventral) e lobus sinister lateralis (território dorsal) $(3,3 \%)$. Nos muares, o ductus principalis sinister apresentase livre de aferentes em seis dos 30 fígados $(20 \%)$ acolhendo, nos outros $24(80 \%)$, contingentes procedentes exclusivamente do lobus quadratus.

Analisando agora os dados encontrados para os muares e os de Marçal et al. (1993) para os eqüinos da raça Puro Sangue Inglês, vale dizer, a sistematização do ductus principalis sinister, observa-se para o ductus principalis sinister, número variável de raízes que, em sucessivos arranjos, compõem as seguintes vias: o ductus medius lobi sinistri lateralis mais ductus ventralis lobi sinistri lateralis, ductus lobi sinistri medialis, o ductus dorsalis lobi sinistri lateralis e o ductus lobi quadrati $(26,7 \%)$ e $(36,7 \%)$, respectivamente; o ductus medius lobi sinistri lateralis mais o ductus ventralis lobi sinistri lateralis, - ductus lobi sinistri medialis, o ductus dorsalis lobi sinistri lateralis (10\%) e (16,7\%), respectivamente; o ductus medius lobi sinistri lateralis mais ductus ventralis lobi sinistri lateralis, - ductus dorsalis lobi sinistri lateralis, o ductus lobi sinistri medialis e o ductus lobi quadrati $(30 \%)$ e $(6,7 \%)$, respectivamente; o ductus medius lobi sinistri lateralis mais ductus ventralis lobi sinistri lateralis, ductus dorsalis lobi sinistri lateralis, ducius lobi sinistri medialis $(3,3 \%)$, em ambos os estudos. Os arranjos que se seguem foram encontrados apenas nos muares: o ductus medius lobi sinistri lateralis mais ductus ventralis lobi sinistri lateralis, ductus lobi sinistri medialis e o ductus lobi quadrati (6,7\%); o ductus medius lobi sinistri lateralis mais ductus ventralis lobi sinistri lateralis, ductus lobi sinistri medialis, ductus lobi quadrati (10\%); o ductus medius lobi sinistri lateralis mais ductus ventralis lobi sinistri lateralis, ductus lobi sinistri medialis, ductus dorsalis lobi sinistri lateralis, ductus lobi quadrati (3,3\%); o ductus medius lobi sinistri lateralis mais o ductus ventralis lobi sinistri lateralis, ductus lobi sinistri medialis, ductus lobi quadrati, ductus dorsalis lobi sinistri lateralis $(3,3 \%)$.

Por outro lado, cabe salientar que, da mesma forma, as várias combinações e correspondentes freqüências presentes no trabalho de Marçal et al. (1993) e ausentes em muares, mais precisamente: o ductus ventralis lobi sinistri lateralis mais 
ductus lobi sinistri medialis, o ductus medius lobi sinistri lateralis, o ductus lobi sinistri lateralis e o ductus lobi quadrati $(13,3 \%)$; o ductus ventralis lobi sinistri lateralis mais ductus lobi sinistri medialis, o ductus dorsalis lobi sinistri lateralis mais ductus medius lobi sinistri lateralis e o ductus lobi quadrati $(3,3 \%)$; o ductus ventralis lobi sinistri lateralis mais o ductus lobi sinistri medialis, o ductus medius lobi sinistri lateralis e 0 ductus dorsalis lobi sinistri lateralis (3,3\%); o ductus medius lobi sinistri lateralis mais ductus lobi sinsitri medialis, o ductus dorsalis lobi sinistri lateralis, o ductus ventralis lobi sinistri lateralis e o ductus lobi quadrati (3,3\%); o ductus dorsalis lobi sinistri lateralis mais ductus medius lobi sinistri lateralis mais ductus ventralis lobi sinistri lateralis, o ductus lobi sinistri medialis e o ductus lobi quadrati (3,3\%); o ductus dorsalis lobi sinistri lateralis mais ductus medius lobi sinistri lateralis, 0 ductus ventralis lobi sinistri lateralis e o ductus lobi sinistri medialis $(3,3 \%)$; o ductus medius lobi sinistri lateralis mais ductus ventralis lobi sinistri lateralis, o ductus lobi sinistri medialis, o ductus dorsalis lobi dextri e o ductus lobi quadrati $(3,3 \%)$; o ductus medius lobi sinistri lateralis mais ductus ventralis lobi sinistri lateralis, o ductus lobi sinistri medialis, 0 ductus dorsalis lobi sinistri lateralis, o ductus lobi quadrati e o ductus dorsalis lobi dextri (3,3\%).

O cálculo de porcentagens revelou que a combinação mais freqüente entre os referidos ductos, por ordem é: o ductus

\section{Referências}

BERG, R. Anatomía topográfica y aplicada de los animales domésticos. Madri: Editorial AC, 1978. p. 216.

BOURDELLE, E.; BRESSOU, C. Anatomie régionale des animaux domestiques. Paris: J. B. Baillière et Fils, 1937. v. 1, p. 51.

BRADLEY, O. C. The thorax and abdomen of the horse. Edinburgh: W. Green \& Son, 1922. p. 123 e 124.

BRUNI, A. C.; ZIMMERL, U. Anatomia degli animali domestici. Milano: Francesco Vallardi, 1947. v. 2, p. 84.

CARADONNA, G. B. Apparecchio gastro-pulmonare. In: ZIMMERL, U. Tratatto di anatomia veterinaria. Milano: Francesco Vallardi, 1930. v. 2, p. 563.

CHAVEAU, A.; ARLOING, S. D'anatomie comparée des animaux domestiques. Paris, J. - B. Baillière et Fils, 1903, p. 663.

D'ARCE, R. D.; FLECHTMANN, C. H. W. Introdução à anatomia e fisiologia animal. 2. ed. São Paulo: Nobel, 1989. p. 134.

DUPRAT, P. Segmentation hépatique chez le cheval. Alfort: Au Manuscrit, 1970.115p.

DYCE, K. M.; SACK, W. O.; WENSING, C. J. G. Tratado de anatomia veterinária. Rio de Janeiro: Guanabara Koogan, 1987. p. 355.

ELLENBERGER, W. EBAUM, H. Handbuch dervergleichenden anatomie der haustiere. 17 Auf. Berlin: Julius Springer, 1952. v. 1, p. 438 e 439. FAVILLI, N. L' anatomia des fegato nei principali animali domestici (cavallo, bue, maiale, cane). Pisa, Tip. A. Cesari, 1923. p. 53-59.

FERREIRA, N. Contribuição aos estudos das vias bilíferas intra e extra-hepáticas no Equus caballus. Sistematização do ramus principalis dexter, ramus principalis sinistere do ductus coledocus. Rev. Fac. Med. Vet. Zootec. Univ. S. Paulo, v. 11, p. 201-226, 1974.

FRANDSON, R. D. Anatomia e fisiologia dos animais domésticos. 2. ed. Rio de Janeiro: Guanabara Koogan, 1979. p. 249. medius lobi sinistri lateralis mais ductus ventralis lobi sinistri lateralis, o ductus lobi sinistri medialis, o ductus dorsalis lobi sinistri lateralis e o ductus lobi quadrati, tanto em muares quanto em eqüinos Puro-Sangue Inglês (Marçal et al., 1993).

Para Stopiglia (1981), no eqüino Puro-Sangue Inglês o ductus choledocus, em $46,7 \%$ dos casos, é formado pela união do ductus principalis dexter e ductus principalis sinister, enquanto que nos $53,3 \%$ restantes, pela tríplice convergência do ductus principalis dexter, ductus principalis sinister e ductus ventralis lobi dextri $(36,7 \%)$, ou do ductus principalis dexter, ductus principalis sinister e ductus lobi quadrati $(16,7 \%)$, sendo que nos muares foi observada apenas a união do ductus principalis sinister e do ductus principalis dexter.

\section{Conclusões}

Excluindo-se os afluentes inominados, encontrou-se um modelo padrão concernente ao arranjo dos ductos biliares do fígado de muar (Equus asinus $X$ Equus caballus), melhor explicando: a) o ductus choledocus forma-se da união do ductus principalis dexter e ductus principalis sinister, b) o ductus principalis sinister pelos ductus medius lobi sinistri lateralis mais o ductus ventralis lobi sinistri lateralis, o ductus lobi sinistri medialis, o ductus dorsalis lobi sinistri lateralis e o ductus lobi quadrati.

GETTY, R.; SISSON e GROSSMAN. Anatomia dos animais domésticos. 5. ed. Rio de Janeiro: Interamericana, 1986. v. 1, p. 463.

GONZALEZY GARCIA, J. e GONZALEZ ALVAREZ, R. G. Anatomía comparada de los animales domésticos. 7. ed. Madrid: Gráficas Canales, 1961. p. 486.

INTERNATIONAL COMMITTEEONVETERINARY GROSSANATOMICAL NOMENCLATURE. Nomina anatomica veterinaria. Apparatus digestorius. 4. ed. Zurich, 1994. p. 12.

JABLAN-PANTIC, O. Characteristics and comparative ratios of intraepatic bile duct in domestic animals. Acta vet., Beograd, v. 13, p. 314, 1963.

KRAEHMER, R. e SCHORÖDER, L. Anatomia de los animales domésticos. Zaragoza: Acribia, 1976. p. 203.

MARÇAL, A. V.; FERREIRA, N.; FERNANDESFILHO, A.; STOPIGLIA, A. J. Contribuição ao estudo das vias bilíferas em equinos da raça PuroSangue Inglês. Sistematização do ductus principalis sinister. Rev. Bras. Ciên. Morfol., v. 10, n. 1, p. 22-27, 1993.

NICKEL, R.; SCHUMMER, A.; SEIFERLE, E.; SACK, W. O. The viscera of the domestic mammals. Berlim: Paul Parey, 1973, p. 115.

NUSSHAG, W. Anatomía y fisiología de los animales domésticos. Zaragoza: Acribia, 1977. p. 295.

OTTAVIANI, G. Richerche radiografiche comparative sulle vie bilifere intraepatiche. Atti Ist. Veneto Sci., v. 92, n. 2, p. 10851087, 1933.

SCHWARZE, E. e SCHRÖDER, L. Compendio de anatomía veterinaria. Zaragoza: Acribia, 1970. v. 2, p. 103.

STOPIGLIA, A. J. Contribuição ao estudo das vias biliferas em equinos da raça Puro-Sangue Inglês. Comportamento do ductus choledocus. Sistematização do ramus principalis dexter. São Paulo, 1981. 55 p. Dissertação de mestrado - Faculdade de Medicina Veterinária e Zootecnia-USP. 